\title{
CONSIDERAÇÕES A PROPÓSITO DO TRATAMENTO MEDICAMENTOSO DE 1217 PACIENTES EPILEEPTICOS. I: ESTUDO EM RELAÇÃO AO TIPO DE EPILEPSIA E AO ELETRENCEFALOGRAMA
}

\author{
Luís MARques-ASSIS *
}

Embora a cirurgia da epilepsia venha se desenvolvendo de maneira acentuada, é inegável que o tratamento clínico, com base medicamentosa, prevalece nas indicaçōes. Por outro lado, embora certos tipos de epilepsia apresentem melhor resposta terapêutica que outros, está comprovado que sòmente dará bons resultados o tratamento bem orientado e desenvolvido de maneira continua.

No presente trabalho será estudada a evolução de 1217 casos de epilepsia submetidos a tratamento medicamentoso, relativamente ao tipo de epilepsia e ao padrão eletrencefalográfico.

\section{MATERIAL E METODOS}

Foram estudados 1217 pacientes matriculados no Ambulatório de Epilepsia do Departamento de Neurologia do Hospital das Clínicas de São Paulo. Dêsse total, 675 eram do sexo feminino e 542 do sexo masculino. Em 651 casos (53\%) a idade estava compreendida entre 10 e 30 anos. O tempo de doença variou entre um dia e 30 anos. O exame neurológico foi normal em todos, não havendo sinais de hipertensão intracraniana. Todos os pacientes foram submetidos a eletrencefalograma; em 1192 casos foi feito exame de líquido cefalorraqueano que revelou, em 6 casos, a presença de neurocisticercose.

Quanto às manifestaçôes clínicas, em 220 casos a convulsão era pura, em 776 era acompanhada de aura (em 77 casos havia, concomitantemente, crises psicomotoras), em 146 casos a convulsão ocorria no sono e, em 13, as crises eram de tipo psicomotor; outros tipos de crises ocorreram em 62 casos. Em relação ao eletrencefalograma, em 367 casos êsse exame foi normal, em 699 casos apresentou anormalidade focais e, em 151 casos, anormalidades difusas. Dentre as anormalidades focais, em 487 casos havia disritmia temporal, uni ou bilateral; dentre as difusas, em 52 casos a anormalidade era paroxística, bilateral e sincrona.

No tratamento foram utilizados, isoladamente ou em associação, barbitúricos, hidantoinatos, primidonas e trimetadione. Em alguns casos foram utilizados, como adjuvantes, a clorpromazina, butirofenona, benzodiazepinicos e acetazolamida. $O$ tempo de tratamento variou entre um ano e 7 anos e 4 meses. Foram conside-

Departamento de Neurologia, Faculdade de Medicina da Universidade de São Paulo (Prof. Adherbal Tolosa): * Assistente. 
rados melhorados os casos que tiveram diminuida a freqüência das crises; a melhora foi considerada sensivel quando a frequiência caiu para a metade ou menos; foram considerados em remissão os casos em que cessaram por completo todos os tipos de crises.

Os resultados, em índices percentuais, podem ser observados nos quadros 1 a 5 .

\section{COM E N TA R I OS}

Embora as epilepsias, dada a sua freqüência e pelas implicações médicas e sociais graves que podem determinar, venham sendo de longa data exaustivamente estudadas, as referências bibliográficas relativas ao prognóstico dessa afecção, especialmente em relação ao tipo das crises, são surpreendentemente raras. Clàssicamente é admitido que as epilepsias temporais são as mais resistentes ao tratamento, especialmente aquelas com tradução clínica de tipo psicomotor. Mesmo nos tratados que versam sôbre o assunto 4,5,6, a matéria tem sido abordada de maneira superficial.

Strobos $^{7}$ (1959) estudou a evolução de 228 pacientes epilépticos em relação ao padrão eletrencefalográfico; o autor justificou não ter levado em conta as manifestaçōes clínicas na investigação pela variabilidade dos sintomas, quando considerados doentes diferentes, e pela existência, com certa freqüência, de crises inespecíficas; em relação ao EEG, encontrou melhores resultados do tratamento no grupo de pacientes com disritmia paroxística bilateral e síncrona; os piores resultados foram obtidos nos casos com disritmias hemisféricas; o melhor prognóstico, entre as disritmias focais, coube ao grupo com focos centroparietais. Arieff 1 (1951) estudou 311 pacientes epilépticos internados em hospital especialisado, no período entre 1935 e 1948; o autor utilizou, para o tratamento, brometos, fenobarbital, difenilidantoína e feniletilmetilidantoína, isoladamente ou em associação; obteve maior índice de remissão quando a crise convulsiva estava presente no quadro; o indice de remissão caía para 56\% quando havia crises de tipo pequeno mal e "equivalentes". Yahr e col. ${ }^{8}$ (1952), estudando 319 casos de epilepsia de natureza indeterminada ou conseqüente a tumor, traumatismo, infecções ou doenças degenerativas, chegou a conclusōes semelhantes: melhor resposta terapêutica dos pacientes com crises focais e com convulsões enquanto que, naqueles com crises mistas, a chance do contrôle da doença caía de quase $50 \%$. Bird e col. (cit. por Penfield e Jasper ${ }^{6}$ ) estudaram 257 epilépticos adultos do Royal Victoria Hospital; o menor índice de remissões foi obtido no grupo focal $(21,8 \%)$, especialmente temporal $(18,3 \%)$ em relação as formas idiopáticas e não localizadas. Fukuyama e col. ${ }^{2}$ (1963) estudaram um grupo de 801 crianças com epilepsia obtendo os melhores resultados nas crises de "ausência" (88\% de respostas favoráveis); nas crianças com convulsões, melhoras ocorreram em $68 \%$ dos casos; nas com crises autônomas, em 64\%; na experiência dos autores as crises mioclônicas e as psicomotoras foram as de mais difícil contrôle. O estudo eletrencefalográfico foi feito em relação à presença ou não de espículas nos traçados. 


\begin{tabular}{|c|c|c|c|c|c|c|c|c|c|c|}
\hline \multirow{2}{*}{$\begin{array}{l}\text { Manifestaçōes } \\
\text { clínicas }\end{array}$} & \multicolumn{2}{|c|}{ I } & \multicolumn{2}{|c|}{ Md } & \multicolumn{2}{|c|}{ Ms } & \multicolumn{2}{|c|}{$\mathbf{R}$} & \multirow{2}{*}{$\frac{M s+R}{\%}$} & \multirow{2}{*}{ Total } \\
\hline & N. ${ }^{\circ}$ & $\%$ & N.० & $\%$ & N.o & $\%$ & N.o & $\%$ & & \\
\hline GM pura & 23 & 10,4 & 14 & 6,4 & 80 & 36,4 & 103 & 46,8 & 83,2 & 220 \\
\hline GM com aura & 48 & 6,2 & 33 & 4,2 & 403 & 51,9 & 292 & 37,7 & 89,6 & 776 \\
\hline GM no sono & 10 & 6,8 & 6 & 4,1 & 44 & 30,1 & 86 & 59,0 & 89,1 & 146 \\
\hline Automatismo & 1 & 7,8 & 0 & 0 & 6 & 46,1 & 6 & 46,1 & 92,2 & 13 \\
\hline Outras crises & 5 & 8,1 & 2 & 3,2 & 25 & 40,3 & 30 & 48,4 & 88,7 & 62 \\
\hline
\end{tabular}

Quadro 1 -Evolução de 1.217 pacientes epilépticos em relação às manifestações clinicas: $I$ = inalterado; Md = melhora discreta; $M s=$ melhora sensivel; $R=$ remissão. 


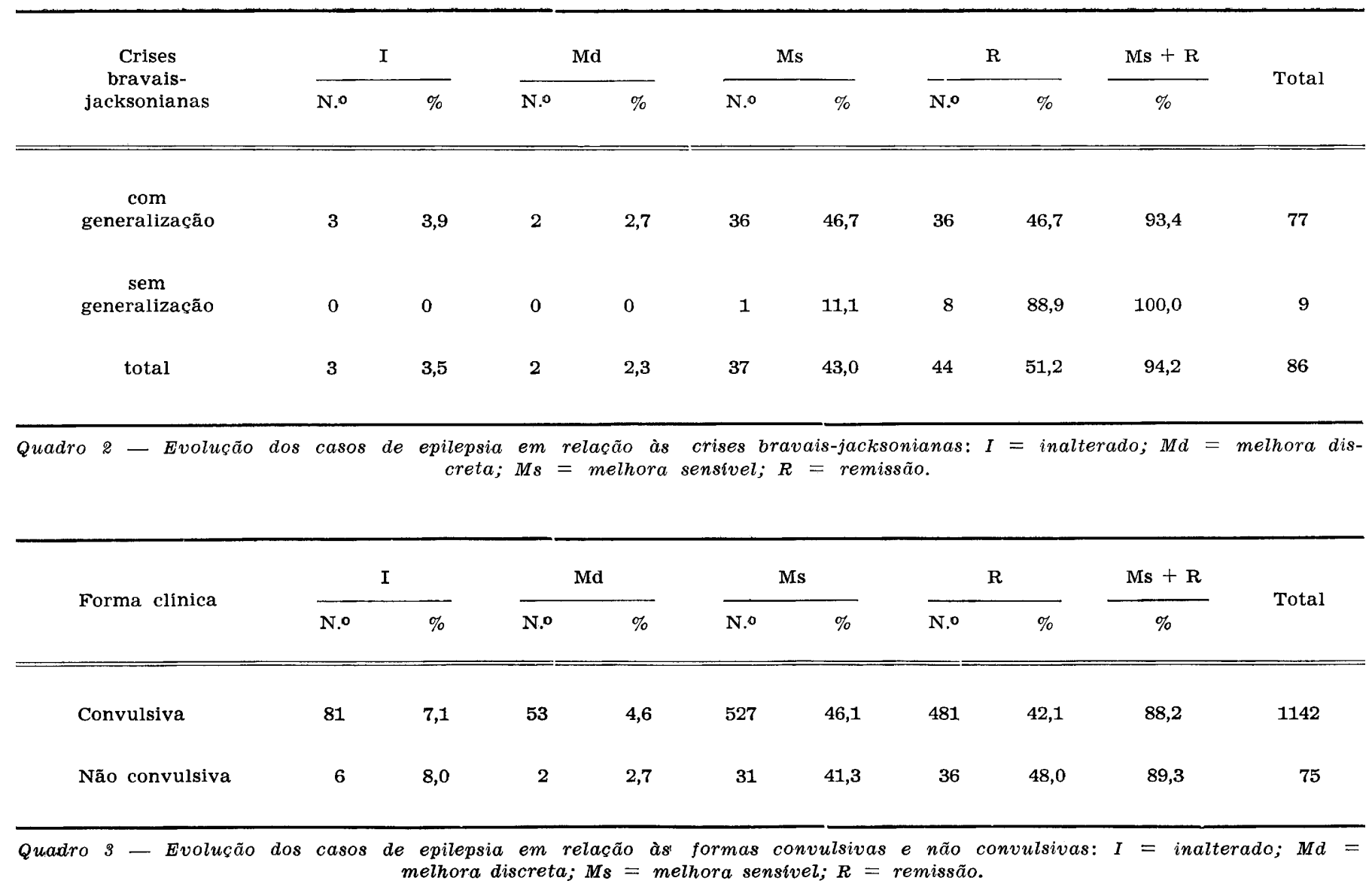




\begin{tabular}{|c|c|c|c|c|c|c|c|c|c|c|}
\hline \multirow{2}{*}{ EEG } & \multicolumn{2}{|c|}{ I } & \multicolumn{2}{|c|}{$\mathbf{M d}$} & \multicolumn{2}{|c|}{ Ms } & \multicolumn{2}{|c|}{$\mathrm{R}$} & \multirow{2}{*}{$\frac{\mathrm{Ms}+\mathrm{R}}{\%}$} & \multirow{2}{*}{ Total } \\
\hline & N.o & $\%$ & N.o & $\%$ & N.o & $\%$ & N.o & $\%$ & & \\
\hline Normal & 22 & 6,0 & 13 & $\mathbf{3 , 5}$ & 162 & 44,1 & 170 & 46,4 & 90,5 & 367 \\
\hline Focal & 51 & 7,3 & 34 & 4,9 & 331 & 47,3 & 283 & 40,5 & 87,8 & 699 \\
\hline Difuso & 14 & 9,3 & 8 & 5,3 & 65 & 43,0 & 64 & 42,4 & 85,4 & 151 \\
\hline
\end{tabular}

Quadro 4 - Evolução dos casos de epilepsia em relação ao eletrencefalograma: $I=$ inalterado; $M$ d = melhora discreta; $M s=$ melhora sensivel; $R=$ remissão.

\begin{tabular}{|c|c|c|c|c|c|c|c|c|c|c|}
\hline \multirow{2}{*}{ Disritmia } & \multicolumn{2}{|c|}{$\mathrm{I}$} & \multicolumn{2}{|c|}{ Md } & \multicolumn{2}{|c|}{ Ms } & \multicolumn{2}{|c|}{$\mathbf{R}$} & \multirow{2}{*}{$\frac{\mathrm{Ms}+\mathrm{R}}{\%}$} & \multirow{2}{*}{ Tota1 } \\
\hline & N.o & $\%$ & N.o & $\%$ & N.o & $\%$ & N.o & $\%$ & & \\
\hline Temporal $\mathbf{E}$ & 18 & 6,6 & 14 & 5,1 & 133 & 48,9 & 107 & 39,4 & 88,3 & 272 \\
\hline Temporal D & 6 & 6,2 & 4 & 4,2 & 57 & 59,4 & 29 & 30,2 & 89,6 & 96 \\
\hline Bitemporal & 12 & 10,1 & 7 & $\mathbf{5 , 9}$ & 53 & 44,5 & 47 & 39,5 & 84,0 & 119 \\
\hline Total & 36 & 7,4 & 25 & 5,1 & 243 & 49,9 & 183 & 37,6 & 87,5 & 487 \\
\hline
\end{tabular}

Quadro 5 - Evolução dos casos de epilepsia em relaçāo às disritmias temporais: $I$ = inalterado; $M$ d $=$ melhora discreta; $M s=$ melhora sensivel; $R=$ remissão. 
Juul-Jensen (cit. por Gundmundson ${ }^{3}$ ) estudou 1008 casos de epilepsia; nos casos com sintomas de epilepsia temporal o autor observou $35,6 \%$ de remissões; nos demais, $51,9 \%$. O prognóstico era melhor nos pacientes com crises GM $(63,5 \%)$, sendo pior quando ocorriam vários tipos de crises combinadamente $(42,0 \%$ em associação de crises GM com focal e $34,1 \%$ em associação de crises GM com "crises menores"). Gundmundson ${ }^{3}$ estudou 987 casos, concluindo ser o prognóstico melhor nos doentes com crises GM e pior nos casos com crises combinadas.

Nossos estudos foram desenvolvidos em 1217 casos de epilepsia, nos quais predominaram nìtidamente pacientes com idade superior a 10 anos. No que se refere ao tipo de crise, conforme se pode observar no quadro 1 , o grupo que apresentou pior resposta terapêutica foi o dos pacientes com crises de tipo GM puro, de origem provável na região centrencefálica; por outro lado, as melhores respostas foram verificadas, contràriamente ao que tem sido referido, nos pacientes que apresentavam crises exclusivamente psiccmotoras; no entanto, o pequeno número de casos dêsse grupo impede que possam ser tiradas conclusões definitivas. Deve-se notar que dos $\mathbf{7 7 6}$ casos que apresentaram convulsões secundárias, em 77 casos havia, associadamente, crises de tipo psicomotor; nesse grupo (4 permaneceram inalterados $-5,2 \%, 3$ apresentaram melhoras discretas $-3,9 \%, 52$ apresentaram melhoras sensiveis - 67,5\% e 18 apresentaram remissão da sintomatologia - 23,4\%), estavam os pacientes com menor índice percentual de remissões $(23,4 \%)$. A observação do quadro 1 permite verificar, ainda, que maior índice de remissão foi obtido nos casos com convulsão noturna $(59,0 \%)$ enquanto que o menor índice $(37,7 \%)$ foi observado nas formas convulsivas secundárias.

No quadro 2 está sintetizado o estudo da evolução em relação às crises de tipo bravais-jaksoniano; pode-se verificar que os resultados obtidos com o tratamento dêsse grupo foram excelentes, com $51,2 \%$ de remissões e com $94,2 \%$ de resultados favoráveis. A comparação dos grupos de pacientes que apresentaram crises bravais-jacksonianas com e sem generalização mostra melhores resultados terapêuticos nos casos em que não houve generalização ( $88,9 \%$ de remissões contra $46,7 \%$ nos casos com generalização da crise).

Finalmente, a comparação das formas convulsivas da epilepsia (quadro 3) com as formas não convulsivas, não mostrou diferenças significativas, embora fossem obtidos maiores indices de remissões nas formas não convulsivas $(48,0 \%)$ em relação às convulsivas $(42,1 \%)$.

Quanto à evolução dos pacientes submetidos a tratamento medicamentoso, relativamente ao padrão eletrencefalográfico, a análise dos quadros 4 e 5 mostram alguns dados que podem ser avaliados, tendo em vista os obtidos do estudo das manifestaçōes clínicas. Assim é que, conforme era de se esperar, encontramos maiores índices de formas incontroláveis entre os pacientes com EEG com anormalidade difusa; por outro lado, maior índice percentual de evolução favorável foi verificado nos casos com EEG de tipo focal. Deve-se notar que os casos com disritmia difusa de tipo paroxístico, bilateral e síncromo, caracterizando o grupo das epilepsias cen- 
trencefálicas de modo geral, tiveram evolução melhor (5 permaneceram inalterados $-9,6 \%, 8$ apresentaram melhoras discretas $-2,0 \% ; 24$ apresentaram melhoras sensiveis - 46,1\% e 22 apresentaram remissão da sintomatologia - 42,3\%), fazendo supor que os casos de pior prognóstico sejam aquêles com anormalidades eletrencefalográficas difusas, traduzindo a exteriorização de foco profundo, subcortical, mas não localizado no centrencéfalo pròpriamente dito. Além disso, sendo o grupo de pacientes por nós estudado constituído quase que exclusivamente de adultos, pode-se compreender fàcilmente o pequeno número de casos com disritmia centrencefálica com tradução clínica de tipo ausência simples, de contrôle terapêutico relativamente simples ${ }^{2}$; êsse fato auxilia também na comparação de nossos resultados relativamente aos referidos na literatura. De qualquer forma, êsses achados corroboram os resultados clínicos obtidos no presente trabalho, segundo os quais o prognóstico é pior justamente nas formas convulsivas puras, em relação às secundárias. Por outro lado é perfeitamente compreensível que os melhores resultados terapêuticos tenham sido obtidos nos casos com EEG normal; além das formas mais benigmas da epilepsia se congregarem nesse grupo, deve ser levado em conta o fato das epilepsias bravaisjacksonianas, de bom prognóstico, como vimos, geralmente não terem tradução eletrencefalográfica.

O estudo da evolução dos pacientes com disritmia temporal (quadro 5) mostra que, nos casos com anormalidade em ambos os lobos temporais foi maior o número de casos incontroláveis. O menor índice de remissão foi obtido nos casos com foco temporal direito. O estudo da evolução relativamente aos pacientes com epilepsia temporal, de modo geral, permitiu verificar a existência de menor índice de remissão que nas outras formas estudadas $(37,6 \%)$; no entanto, foi obtida evolução favorável em $87,5 \%$ dos casos, o que não deixa de ser um resultado satisfatório.

De qualquer forma, independentemente do tipo de manifestação clínica ou do padrão eletrencefalográfico que o paciente apresente, êste trabalho demonstra que, uma vez instituída a terapêutica correta, com a utilização apenas dos medicamentos mais conhecidos e difundidos em nosso meio, bons resultados podem ser alcançados, podendo-se admitir contrôle satisfatório da moléstia em quase $90 \%$ dos casos.

\section{RESUMO E CONCLUSŌES}

Foram estudados 1.217 pacientes epilépticos, adultos em sua maioria, sem alterações neurológicas focais e sem sinais de hipertensão intracraniana. Foram utilizados, para o tratamento, apenas os medicamentos mais conhecidos e difundidos em nosso meio. O estudo foi feito em relação ao tipo de epilepsia que os pacientes apresentavam e ao padrão eletrencefalográfico.

Quanto à evolução relativamente ao tipo de epilepsia, as investigações desenvolvidas permitiram ao autor chegar às seguintes conclusões: 1) a evolução foi melhor nos pacientes que apresentavam como única manifes- 
tação crises bravais-jacksonianas $(94,2 \%)$ e crises psicomotoras (92,2\%); 2) maior índice percentual de remissōes foi obtido nos pacientes com convulsões no sono $(59,0 \%)$; 3) os pacientes com convulsōes GM puras foram os mais resistentes ao tratamento e aquêles que em maior grau permaneceram incontroláveis; 4) os pacientes com convulsões secundários a descarga focal foram os que mais acentuadamente melhoraram com o tratamento, embora fossem aquêles que apresentassem menor índice percentual de remissão; 5) de tôdas as formas de epilepsia as de tipo bravais-jacksoniano foram as que melhor responderam ao tratamento, especialmente as formas que não se generalizaram; 6) não houve diferenças notáveis, quanto à evolução, quando comparadas as formas convulsivas e não convulsivas da epilepsia.

Em relação à evolução relativamente ao padrão eletrencefalográfico, o autor chegou às seguintes conclusões: 1) a evolução foi melhor nos casos com EEG normal; 2) comparados os casos com EEG anormal, aquêles com disritmia paroxística bilateral e síncrona evoluíram melhor que os com anormalidades difusas de modo geral e que os focais; 3 ) os pacientes com foco temporal tiveram menor índice de remissão que os demais; 4) os casos com foco temporal único (direito ou esquerdo) evoluíram melhor que os com disritmia temporal bilateral; 5) os pacientes com foco temporal direito foram os que apresentaram, percentualmente, menor índice de remissão.

\section{S U M M A R Y}

\section{Drug treatment of 1.217 epileptic patients: I - Study regarding the clinical manifestations and eletroencephalographic patterns.}

The evolution of 1.217 epileptic patients submitted to antiepileptic drug treatment is studied. Barbiturates, hydantoin, primidone and trimethadione were administered to the patients, isolated or in association. The study was made in regard to the clinical manifestations and electroencephalographic pattern.

Concerning the type of seizures, the author draws the following conclusions: 1) the evolution was better in patients with Bravais-Jackson seizures; 2) the highest percentual rate of remission was shown by patients with sleep seizures $(59,0 \%)$; 3) the patients with "grand mal" seizures without aura were the most refractory to the treatment; 4) the patients with generalized fits secondary to focal discharges showed the best results, although evidencing lowest remission index; 5) among all types of seizures, the Bravais-Jacsonian fits showed better evolution, specially the cases without generalization; 6) significant differences of evolution were not found between convulsive and non convulsive epilepsy.

The electroencephalographic study led the author to the following conclusions: 1) the evolution was better in cases with normal EEG; 2) the evolution was better in cases with paroxystic, bilateral and synchronic dysrhythmia than in cases with diffuse abnormalities in sensu latu and with 
focal abnormalities; 3) the patients with temporal dysrhythmia showed the lowest remission index; 4) the patients with unilateral temporal dysrhythmia showed better evolution than those with bilateral temporal dysrhythmia; 5) the patients with right temporal dysrhythmia presented the lowest remission index.

\section{REFEREN CIAS}

1. ARIEF, A. J. - Twelve-year resume in a Clinic for Epilepsy. Dis. nerv. System $12: 19,1951$.

2. FUKUYAMA, Y.; ARIMA, M.; NAGAHATA, M. \& OKADA, R. - Medical treatment of epilepsies in childhood; a long-term survey of 801 patients. Epilepsia $4: 207,1963$.

3. GUNDMUNDSSON, G. - Epilepsy in Iceland. A clinical and epidemiological investigation. Acta neurol. scand. supl. 25, 1966.

4. LENNOX, W. G. \& LENNOX, M. A. - Epilepsy and Related Disorders. Little-Brown Co., Boston, 1960.

5. LIVINGSTONE, S. - Convulsive Disorders in Children. Charles C. Thomas, Springfield (Illinois), 1954.

6. PENFIELD, W. \& JASPER, H. - Epilepsy and the Functional Anatomy of the Human Brain. Little-Brown Co., Boston, 1954.

7. STRobos, R. J. - Prognosis in convulsive disorders. Arch. Neurol. (Chicago) $1: 216,1959$.

8. YAHR, M. D.; SCIARRA, D.; CARTER, S. \& MERRIT, H. H. - Evaluation of standart anticonvulsant therapy in three hundred nineteen patients. JAMA 150:663, 1952 .

Departamento de Neurologia - Faculdade de Medicina da Universidade de São Paulo - Caixa Postal 3461 - São Paulo, SP - Brasil. 